\title{
KLT-type relations for QCD and bicolor amplitudes from color-factor symmetry
}

\author{
Robert W. Brown ${ }^{a}$ and Stephen G. Naculich ${ }^{b}$ \\ ${ }^{a}$ Department of Physics, Case Western Reserve University, \\ Cleveland, OH 44106 U.S.A. \\ ${ }^{b}$ Department of Physics, Bowdoin College, \\ Brunswick, ME 04011 U.S.A. \\ E-mail: rwb@case.edu, naculich@bowdoin.edu
}

ABSTRACT: Color-factor symmetry is used to derive a KLT-type relation for tree-level QCD amplitudes containing gluons and an arbitrary number of massive or massless quarkantiquark pairs, generalizing the expression for Yang-Mills amplitudes originally postulated by Bern, De Freitas, and Wong. An explicit expression is given for all amplitudes with two or fewer quark-antiquark pairs in terms of the (modified) momentum kernel.

We also introduce the bicolor scalar theory, the "zeroth copy" of QCD, containing massless biadjoint scalars and massive bifundamental scalars, generalizing the biadjoint scalar theory of Cachazo, He, and Yuan. We derive KLT-type relations for tree-level amplitudes of biadjoint and bicolor theories using the color-factor symmetry possessed by these theories.

KEYwords: Duality in Gauge Field Theories, Gauge Symmetry, Perturbative QCD, Scattering Amplitudes

ARXIV EPRINT: 1802.01620 


\section{Contents}

1 Introduction 1

2 Bicolor scalar theory 3

3 Color-factor symmetry of the bicolor scalar theory 9

$4 \quad$ KLT-type relations for biadjoint and bicolor amplitudes $\quad 11$

$5 \quad$ KLT-type relations for QCD (and gravity) amplitudes 15

$\begin{array}{lll}5.1 & \text { Derivation of QCD KLT-type relations using color-factor symmetry } & 15\end{array}$

$\begin{array}{lll}5.2 & \text { Derivation of QCD KLT-type relations using generalized gauge invariance } & 17\end{array}$

$\begin{array}{lll}5.3 & \text { Gravitational KLT amplitudes } & 18\end{array}$

$\begin{array}{llr}6 & \text { Conclusions } & 19\end{array}$

A Color-factor shifts for five- and higher-point amplitudes 20

\section{Introduction}

Over thirty years ago, Kawai, Lewellen, and Tye (KLT) discovered that tree-level closedstring scattering amplitudes can be expressed as a sum of products of open-string scattering amplitudes [1]. In the field-theory limit, the KLT formula relates gravitational scattering amplitudes to products of gauge-theory scattering amplitudes [2-9]. The tree-level $n$ graviton amplitude may be written in the compact form

$$
\mathcal{A}_{n}^{\text {grav }}=-\sum_{\sigma, \tau \in S_{n-3}} A(1,3, \sigma, 2) S[\sigma \mid \tau]_{3} A(2,3, \tau, 1)
$$

where $A(\cdots)$ denotes color-ordered (or partial) $n$-gluon amplitudes, $S[\cdots]$ is the momentum kernel (see eq. (4.9) below for the explicit definition) and $\sigma, \tau$ range over all permutations of $\{4, \cdots, n\}$. The field-theory formula (1.1) was a harbinger of more recent developments showing that tree-level gravitational amplitudes can be obtained as a double copy of gauge-theory amplitudes by replacing the color factors $c_{i}$ that appear in a cubic decomposition of gauge-theory amplitudes with kinematic numerators $\tilde{n}_{i}$ that obey the same algebraic relations [10,11]. The classical spacetime background itself can be constructed through a double-copy procedure [12-16]. Much evidence has accumulated [17-28] for the conjecture $[10,17]$ that color-kinematic duality and the double-copy procedure also apply to the integrands of loop-level amplitudes, but difficulties remain [29, 30]. Quite recently, the KLT formula itself has been generalized to one-loop gravitational amplitudes [31, 32]. 
In 1999, Bern, De Freitas, and Wong [33] proposed an expression analogous to eq. (1.1) for the color-encoded tree-level $n$-gluon amplitude

$$
\mathcal{A}_{n}^{\text {gluon }}=-\sum_{\sigma, \tau \in S_{n-3}} A(1,3, \sigma, 2) S[\sigma \mid \tau]_{3} A^{(\mathrm{s})}(2,3, \tau, 1)
$$

in terms of partial gauge-theory amplitudes $A(\cdots)$ and dual partial scalar amplitudes $A^{(\mathrm{s})}(\cdots)$, which are obtained from partial gauge-theory amplitudes by replacing kinematic numerators $n_{i}$ with color factors $c_{i}$ [11]. Subsequently proven in ref. [34] using BCFW recursion relations [35], eq. (1.2) makes manifest that a subset of $(n-3)$ ! of the partial amplitudes is sufficient to produce the full color-encoded amplitude. ${ }^{1}$ Consequently, the full set of partial amplitudes $A(\cdots)$ can be expressed in terms of these $(n-3)$ ! independent partial amplitudes $A(1,3, \sigma, 2)$; these are the well-known Bern-Carrasco-Johansson (BCJ) relations [10].

In addition to being gauge invariant, tree-level gauge-theory amplitudes have been shown to possess a color-factor symmetry [39, 40]. For each external gluon in the amplitude, there is a family of momentum-dependent shifts of the color factors $c_{i}$ that leave the amplitude invariant. These shifts are analogous to generalized gauge transformations of the kinematic numerators $n_{i}$ but more restrictive because they preserve the Jacobi identities satisfied by color factors (whereas generalized gauge transformations can relate Jacobisatisfying kinematic numerators to non-Jacobi-satisfying kinematic numerators). The dual partial amplitudes $A^{(\mathrm{s})}(\cdots)$ appearing in eq. (1.2), which depend on the color factors $c_{i}$, are themselves invariant under color-factor shifts. Thus eq. (1.2) represents a decomposition of the amplitude in terms of building blocks that are simultaneously gauge invariant and color-factor symmetric.

In this paper we use the color-factor symmetry of gauge-theory amplitudes to provide an alternative derivation of the KLT-type formula (1.2) for the $n$-gluon amplitude. As a by-product, we obtain a new KLT-type formula for the biadjoint scalar theory [9]

$$
\mathcal{A}_{n}^{\text {biadjoint }}=-\sum_{\sigma, \tau \in S_{n-3}} \tilde{A}^{(\mathrm{s})}(1,3, \sigma, 2) S[\sigma \mid \tau]_{3} A^{(\mathrm{s})}(2,3, \tau, 1)
$$

a theory which also possesses color-factor symmetry [39].

We also obtain new KLT-type relations for tree-level $n$-point QCD amplitudes $\mathcal{A}_{n, k}^{\text {qcd }}$ containing $k$ differently flavored quark-antiquark pairs and $n-2 k$ gluons. Tree-level QCD amplitudes can be expressed in terms of partial amplitudes that obey group-theory relations [41-43] as well as (for amplitudes containing gluons) BCJ relations [44]. Johansson and Ochirov (JO) used these relations to define an independent basis of partial amplitudes $A(1, \gamma, 2)$, where $\gamma$ denotes a particular subset (described in the main body of this paper) of permutations of the remaining labels ${ }^{2}$ of quarks and gluons $\{3, \cdots, n\}$. For two or fewer quark-antiquark pairs, the number of independent amplitudes is $(n-3)$ !, and the JO basis

\footnotetext{
${ }^{1}$ The relations (1.2) can be viewed as a generalization of the factorization observed in refs. [36-38].

${ }^{2}$ Here $\{2,4, \cdots, 2 k\}$ denote the labels of (differently flavored) quarks, $\{1,3, \cdots, 2 k-1\}$ the labels of the corresponding antiquarks, and $\{2 k+1, \cdots, n\}$ the labels of gluons.
} 
is simply given by $A(1,3, \sigma, 2)$, where $\sigma$ is any permutation of $\{4, \cdots, n\}$. We establish that, in this case, the color-encoded QCD amplitude can be expressed as

$$
\mathcal{A}_{n, k \leq 2}^{\mathrm{qcd}}=-\sum_{\sigma, \tau \in S_{n-3}} A(1,3, \sigma, 2) S[\sigma \mid \tau]_{3} A^{(\mathrm{s})}(2,3, \tau, 1)
$$

where $A(1,3, \sigma, 2)$ are QCD partial amplitudes, $A^{(\mathrm{s})}(2,3, \tau, 1)$ are corresponding dual partial amplitudes, and $S[\sigma \mid \tau]_{3}$ is the same momentum kernel that appears in the all-gluon expression (1.2), modified by masses in the case of $k=2$ amplitudes. For $k>2$, the number of independent amplitudes in the JO basis is $(n-3) !(2 k-2) / k$ ! [44], and the QCD amplitude can be expressed as

$$
\mathcal{A}_{n, k}^{\mathrm{qcd}}=\sum_{\gamma, \delta \in \mathrm{JO}} A(1, \gamma, 2) T(1 \gamma 2 \mid 2 \delta 1) A^{(\mathrm{s})}(2, \delta, 1)
$$

where $\gamma$ and $\delta$ both belong to the JO set of permutations, and $T(\cdots)$ is the inverse of a particular submatrix of double-partial amplitudes defined later in the paper. Although we do not present an explicit expression for $T(\cdots)$, we conjecture that it can be expressed as an $(n-3)^{\text {th }}$ degree polynomial of kinematic invariants, similar to the momentum kernel. We also write analogous expressions for gravitational scattering amplitudes. Earlier work on extensions of KLT relations to more general gravitational amplitudes includes refs. [33, 45-47].

This paper is structured as follows: in section 2, we introduce the bicolor scalar theory, containing both biadjoint and bifundamental fields. We describe the Melia basis of partial amplitudes, the Melia-Johansson-Ochirov decomposition of the bicolor amplitude, and finally the double-partial amplitudes of the bicolor theory. We end with a KLT-type relation for amplitudes containing only bifundamental fields. In section 3 , we show that the color-factor symmetry possessed by the bicolor theory can be used to derive the null vectors of the matrix of double-partial amplitudes, which leads to BCJ relations for the bicolor partial amplitudes. In section 4, we use the color-factor symmetry to obtain a KLTtype relation for arbitrary bicolor amplitudes. In section 5, we derive KLT-type relations for QCD amplitudes. We also discuss more general gravitational KLT relations. Section 6 contains our conclusions.

\section{Bicolor scalar theory}

The biadjoint scalar theory, introduced by Cachazo, He, and Yuan in ref. [9], is a theory

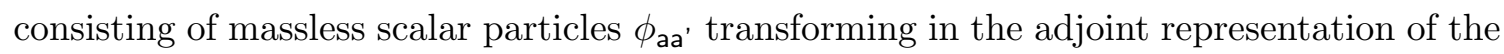
color group $\mathrm{U}(N) \times \mathrm{U}(\tilde{N})$ with cubic interactions of the form

$$
f^{\mathrm{abc}} \tilde{f}^{\mathrm{a} \mathrm{b}^{\prime} \mathrm{c}^{\prime} \phi_{\mathrm{aa}}{ }^{\prime} \phi_{\mathrm{bb}}{ }^{\prime} \phi_{\mathrm{cc}}}
$$

where $f^{\text {abc }}$ and $\tilde{f}^{\mathrm{a}^{\prime} \mathrm{b}^{\prime} \mathrm{c}^{\prime}}$ are the structure constants of $\mathrm{U}(N)$ and $\mathrm{U}(\tilde{N})$. Whereas gravity is a double copy of gauge theory (replacing color factors $c_{i}$ with kinematic numerators $\tilde{n}_{i}$ ), the biadjoint theory can be viewed as a zeroth copy of gauge theory (replacing kinematic 
numerators $n_{i}$ with color factors $\tilde{c}_{i}$ ). The double-partial amplitudes of the biadjoint theory, which depend only on kinematic invariants of the external momenta (without the complications of spin), provide the cleanest examples of amplitudes obeying Kleiss-Kuijf [41] and BCJ relations. The biadjoint theory is also color-factor symmetric [39], which we will use in section 4 to derive KLT-type relations (1.3) for its amplitudes.

Because our goal is also to obtain KLT-type relations for QCD amplitudes containing quarks as well as gluons, we generalize the biadjoint scalar theory to include, in addition to the massless biadjoint fields, several flavors ${ }^{3}$ of (possibly massive) scalar fields $\psi_{(s)}^{\mathrm{ii}}$, $s=1, \cdots, N_{f}$, transforming in the $R \otimes \tilde{R}$ representation of $\mathrm{U}(N) \times \mathrm{U}(\tilde{N})$ with mass terms

$$
m_{(s)}^{2} \bar{\psi}_{(s) \mathrm{ii}} \psi_{(s)}^{\mathrm{ii}}
$$

as well as cubic couplings

$$
\left(T^{\mathrm{a}}\right)^{\mathrm{i}}{ }_{\mathrm{j}}\left(\tilde{T}^{\mathrm{a}^{\prime}}\right)^{\mathrm{i}^{\prime}}{ }^{\prime} \bar{\psi}_{(s) \mathrm{i}}{ }^{\prime} \phi_{\mathrm{aa}}{ }^{\prime} \psi_{(s)}^{\mathrm{j} \mathrm{j}^{\prime}}
$$

where $\left(T^{\mathrm{a}}\right)^{\mathrm{i}}{ }_{\mathrm{j}}$ and $\left(\tilde{T}^{\mathrm{a}^{\prime}}\right)^{\mathrm{i}^{\prime}}{ }^{\prime}$, are generators in the $R$ and $\tilde{R}$ representations. For convenience in what follows, we will refer to $\psi_{(s)}^{\mathrm{j} \mathrm{j}^{\prime}}$ as bifundamental fields (and $\bar{\psi}_{(s) \mathrm{i}}$ as anti-bifundamental fields), although the representation could be more general. We refer to this as the bicolor scalar theory.

Consider a tree-level $n$-point amplitude with both bifundamental and biadjoint fields

$$
\mathcal{A}_{n, k}^{\text {bicolor }}\left(\bar{\psi}_{1}, \psi_{2}, \bar{\psi}_{3}, \psi_{4}, \cdots, \bar{\psi}_{2 k-1}, \psi_{2 k}, \phi_{2 k+1}, \cdots, \phi_{n}\right)
$$

where external fields $\psi$ in the bifundamental representation have even labels and fields $\bar{\psi}$ in the anti-bifundamental representation have odd labels. We assume that the $\psi_{2 \ell}$ all have different flavors (and possibly different masses), with $\bar{\psi}_{2 \ell-1}$ having the corresponding antiflavor (and equal mass) to $\psi_{2 \ell}$. This amplitude is given by a sum over cubic diagrams

$$
\mathcal{A}_{n, k}^{\text {bicolor }}=\sum_{i \in \text { cubic }} \frac{c_{i} \tilde{c}_{i}}{d_{i}}
$$

where $c_{i}, \tilde{c}_{i}$ are color factors constructed from the cubic vertices (2.1) and (2.3), and $d_{i}$ is the product of massless $\phi$ and massive $\psi$ propagators. The cubic diagrams appearing in eq. (2.5) correspond to a subset of the cubic diagrams appearing in an $n$-point amplitude of biadjoint fields. For example, the five-point amplitude with two pairs of bifundamentals is given by

$$
\mathcal{A}_{5,2}^{\text {bicolor }}\left(\bar{\psi}_{1}, \psi_{2}, \bar{\psi}_{3}, \psi_{4}, \phi_{5}\right)=\sum_{i=1}^{5} \frac{c_{i} \tilde{c}_{i}}{d_{i}}
$$

\footnotetext{
${ }^{3}$ This generalization was considered earlier in ref. [48] for a single flavor of bifundamental scalar. See also ref. [49].
} 

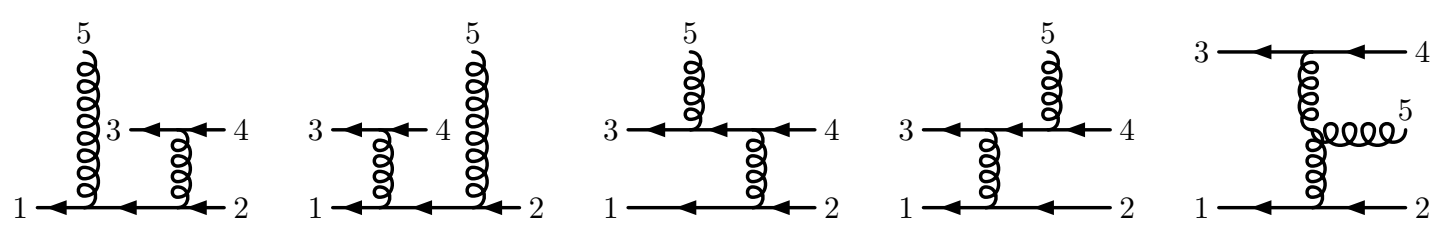

Figure 1. Cubic diagrams $i=1$ through 5 for $\mathcal{A}_{5,2}^{\text {bicolor }}$. Lines with arrows denote bifundamental scalars whereas curly lines denote biadjoint scalars.

where the five contributing cubic diagrams are shown in figure 1, and the color factors and denominators have the form $[39,44]$

$$
\begin{aligned}
& c_{1}=\left(T^{\mathrm{a}_{5}} T^{\mathrm{b}}\right)_{\mathrm{i}_{2}}^{\mathrm{i}_{1}}\left(T^{\mathrm{b}}\right)_{\mathrm{i}_{4}}^{\mathrm{i}_{3}}, \quad d_{1}=\left(s_{15}-m_{1}^{2}\right) s_{34}=2 s_{34} k_{1} \cdot k_{5}, \\
& c_{2}=\left(T^{\mathrm{b}} T^{\mathrm{a} 5}\right)^{\mathrm{i}_{1}}{ }_{\mathrm{i}_{2}}\left(T^{\mathrm{b}}\right)^{\mathrm{i}_{\mathrm{i}_{4}},}, \quad d_{2}=\left(s_{25}-m_{1}^{2}\right) s_{34}=2 s_{34} k_{2} \cdot k_{5}, \\
& c_{3}=\left(T^{\mathrm{b}}\right)^{\mathrm{i}_{1}}{ }_{\mathrm{i}_{2}}\left(T^{\mathrm{a}_{5}} T^{\mathrm{b}}\right)^{\mathrm{i}} \mathrm{i}_{4}, \quad d_{3}=s_{12}\left(s_{35}-m_{3}^{2}\right)=2 s_{12} k_{3} \cdot k_{5}, \\
& c_{4}=\left(T^{\mathrm{b}}\right)^{\mathrm{i}_{1}}{ }_{\mathrm{i}_{2}}\left(T^{\mathrm{b}} T^{\mathrm{a} 5}\right)^{\mathrm{i}_{3}}, \quad d_{4}=s_{12}\left(s_{45}-m_{3}^{2}\right)=2 s_{12} k_{4} \cdot k_{5}, \\
& c_{5}=f^{\mathrm{a} 5 \mathrm{~b} c}\left(T^{\mathrm{b}}\right)^{\mathrm{i}_{1}}\left(T^{c}\right)^{\mathrm{i}_{3}}{ }_{\mathrm{i}_{4}}, \quad d_{5}=s_{12} s_{34}
\end{aligned}
$$

where $s_{i j}=\left(k_{i}+k_{j}\right)^{2}$, with analogous expressions for $\tilde{c}_{i}$. The color factors obey the Jacobi identities

$$
c_{1}-c_{2}+c_{5}=0, \quad c_{3}-c_{4}-c_{5}=0 .
$$

This five-point amplitude will be our prototypical example throughout the paper as it nicely illustrates many of the features of bicolor amplitudes.

Following ref. [9], we define partial amplitudes with respect to each color group factor as well as double-partial amplitudes. The partial amplitude $\tilde{A}^{(\mathrm{s})}(\alpha)$ with respect to the first group factor $\mathrm{U}(N)$, where $\alpha$ denotes an arbitrary permutation of the external particle labels $\{1, \cdots, n\}$, receives contributions from those cubic diagrams $i$ whose color factor $c_{i}$ can be drawn in a planar fashion with the external legs in the cyclic order specified by the permutation $\alpha$. For example, by examining figure 1, one can write down the following five-point partial amplitudes ${ }^{4}$

$$
\tilde{A}^{(\mathrm{s})}(15342)=\frac{\tilde{c}_{1}}{d_{1}}-\frac{\tilde{c}_{3}}{d_{3}}-\frac{\tilde{c}_{5}}{d_{5}}, \quad \tilde{A}^{(\mathrm{s})}(13542)=\frac{\tilde{c}_{3}}{d_{3}}+\frac{\tilde{c}_{4}}{d_{4}}, \quad \tilde{A}^{(\mathrm{s})}(13452)=\frac{\tilde{c}_{2}}{d_{2}}-\frac{\tilde{c}_{4}}{d_{4}}+\frac{\tilde{c}_{5}}{d_{5}}
$$

where the \pm sign in front of each term results from the antisymmetry of the structure constants $f^{\mathrm{abc}}=-f^{\mathrm{bac}}$ and a similar antisymmetry imposed on the generators $\left(T^{\mathrm{a}}\right)^{\mathrm{i}}{ }_{\mathrm{j}}=$ $-\left(T^{\mathrm{a}}\right)_{\mathrm{j}}{ }^{\mathrm{i}}$ (see ref. [44]). In general the partial amplitudes are given by

$$
\tilde{A}^{(\mathrm{s})}(\alpha)=\sum_{i} \frac{M_{i, \alpha} \tilde{c}_{i}}{d_{i}}
$$

where $M_{i, \alpha}$ vanishes if $c_{i}$ does not contribute to $\tilde{A}^{(\mathrm{s})}(\alpha)$, and is otherwise given by 1 or -1 . These partial amplitudes may be regarded as "dual" to color-ordered gauge-theory

\footnotetext{
${ }^{4}$ For the remainder of the paper, we omit the commas between arguments for conciseness.
} 
amplitudes, as they can be obtained from the latter by replacing the kinematic numerators $n_{i}$ with $\tilde{c}_{i}[11]$.

When $k \geq 2$, some of the partial amplitudes vanish because none of the cubic diagrams can contribute. In the five-point amplitude above, for example, $\tilde{A}^{(\mathrm{s})}(13245)$ vanishes because any contributing diagram would require the lines for the differently flavored bifundamental fields to cross, and are thus non-planar. Moreover, there are group-theoretic relations among the nonvanishing bicolor partial amplitudes analogous to the Kleiss-Kuijf [41] and Melia [42, 43] relations among gauge-theory partial amplitudes. For the five-point amplitude, some of the Melia relations are

$$
\begin{aligned}
& \tilde{A}^{(\mathrm{s})}(15432)=-\tilde{A}^{(\mathrm{s})}(15342)-\tilde{A}^{(\mathrm{s})}(13542), \\
& \tilde{A}^{(\mathrm{s})}(14532)=\tilde{A}^{(\mathrm{s})}(13452), \\
& \tilde{A}^{(\mathrm{s})}(14352)=-\tilde{A}^{(\mathrm{s})}(13452)-\tilde{A}^{(\mathrm{s})}(13542) .
\end{aligned}
$$

These group-theoretic relations can be used to define an independent basis ${ }^{5}$ of $(n-2) ! / k$ ! partial amplitudes, called the Melia basis [42, 43].

To describe the Melia basis of partial amplitudes, we recall that a Dyck word of length $2 r$ is a string composed of $r$ letters $\bar{\psi}$ and $r$ letters $\psi$ such that the number of $\bar{\psi}$ 's preceding any point in the string is greater than the number of preceding $\psi$ 's. An easy way to understand this is to visualize $\bar{\psi}$ as a left bracket $\{$ and $\psi$ as a right bracket $\}$, in which case a Dyck word corresponds to a well-formed set of brackets. The number of such words is $(2 r) ! /(r+1) ! r$ ! , the $r^{\text {th }}$ Catalan number. For example for $r=1$ there is only one Dyck word: \{\} , for $r=2$ there are two: \{\}\{\} and $\{\{\}\}$, and for $r=3$ there are five: \{\}\{\}\{\}$,\{\}\{\{\}\}$, $\{\{\}\}\{\},\{\{\}\{\}\}$, and $\{\{\{\}\}\}$. Consider the set of partial amplitudes $A(1, \gamma(3), \cdots, \gamma(n), 2)$, where $\gamma$ is any permutation of $\{3, \cdots, n\}$ such that the set of $k-1 \bar{\psi}$ and $k-1 \psi$ in $\gamma$ form a Dyck word of length $2 k-2$. The biadjoint fields may be distributed anywhere among the $\bar{\psi}$ and $\psi$ in $\gamma$. The number of distinct allowed patterns of $\bar{\psi}, \psi$, and $\phi$ is given by the number of Dyck words of length $2 k-2$ times the number of ways of distributing $n-2 k$ biadjoint fields among the letters of the Dyck word

$$
\frac{(2 k-2) !}{k !(k-1) !} \times\left(\begin{array}{c}
n-2 \\
2 k-2
\end{array}\right) \text {. }
$$

For each allowed pattern, there are $(n-2 k)$ ! distinct choices for the biadjoint labels, and $(k-1)$ ! choices for the $\bar{\psi}$ labels. The label on each $\psi$ is then fixed: it must have the flavor of the nearest unpaired $\bar{\psi}$ to its left. Thus, for example, for $\mathcal{A}_{6,3}^{\text {bicolor }}$ the allowed permutations $\gamma$ are $\bar{\psi}_{3} \psi_{4} \bar{\psi}_{5} \psi_{6}, \bar{\psi}_{5} \psi_{6} \bar{\psi}_{3} \psi_{4}, \bar{\psi}_{3} \bar{\psi}_{5} \psi_{6} \psi_{4}$, and $\bar{\psi}_{5} \bar{\psi}_{3} \psi_{4} \psi_{6}$, whereas for $\mathcal{A}_{5,2}^{\text {bicolor }}$ the allowed permutations are $\phi_{5} \bar{\psi}_{3} \psi_{4}, \bar{\psi}_{3} \phi_{5} \psi_{4}$, and $\bar{\psi}_{3} \psi_{4} \phi_{5}$. Thus the three partial amplitudes given in eq. (2.9) precisely comprise the Melia basis for $\mathcal{A}_{5,2}^{\text {bicolor }}$. The multiplicity of the Melia basis is given by

$$
\frac{(2 k-2) !}{k !(k-1) !} \times\left(\begin{array}{c}
n-2 \\
2 k-2
\end{array}\right) \times(n-2 k) ! \times(k-1) !=\frac{(n-2) !}{k !}
$$

\footnotetext{
${ }^{5}$ The Melia basis amplitudes are independent only with respect to purely group-theoretic relations. As we will see in section 3, color-factor symmetry implies additional (BCJ) relations among these amplitudes.
} 
as found in ref. [43]. We refer to the allowed permutations of $\gamma$ as the Melia set, and the partial amplitudes $A(1 \gamma 2)$ as the Melia basis. For $k=0$ and $k=1$, the elements of $\gamma$ are all biadjoint scalars, the Melia set consists of all permutations of $\{3, \cdots, n\}$, and the Melia basis coincides with the Kleiss-Kuijf basis [41].

Since, for $\gamma$ belonging to the Melia set, the $\tilde{A}^{(\mathrm{s})}(1 \gamma 2)$ form an independent basis of partial amplitudes with respect to the first group factor $\mathrm{U}(N)$, the bicolor amplitude can be written in a proper decomposition [50]

$$
\mathcal{A}_{n, k}^{\text {bicolor }}=\sum_{\gamma \in \text { Melia }} \tilde{A}^{(\mathrm{s})}(1 \gamma 2) C_{1 \gamma 2}
$$

for some set of color factors $C_{1 \gamma 2}$. Equation (2.14) follows from eqs. (2.5) and (2.10), provided that these color factors satisfy

$$
c_{i}=\sum_{\gamma \in \text { Melia }} M_{i, 1 \gamma 2} C_{1 \gamma 2} .
$$

For purely biadjoint amplitudes, the $C_{1 \gamma 2}$ are simply half-ladder color factors

$$
C_{1 \gamma 2} \equiv \sum_{\mathrm{b}_{1}, \ldots, \mathrm{b}_{n-3}} f^{\mathrm{a}_{1} \mathrm{a}_{\gamma(3)} \mathrm{b}_{1}} f^{\mathrm{b}_{1} \mathrm{a}_{\gamma(4)} \mathrm{b}_{2}} \cdots f^{\mathrm{b}_{n-3} \mathrm{a}_{\gamma(n)} \mathrm{a}_{2}}, \quad \gamma \in S_{n-2}, \quad k=0
$$

and eq. (2.14) is the Del Duca-Dixon-Maltoni decomposition [51]. For amplitudes containing one pair of bifundamentals, the $C_{1 \gamma 2}$ are also half-ladder color factors along a bifundamental backbone $[52,53]$

$$
C_{1 \gamma 2}=\left(T^{\mathrm{a}_{\gamma(3)}} T^{\mathrm{a}_{\gamma(4)}} \cdots T^{\mathrm{a}_{\gamma(n)}}\right)^{\mathrm{i}_{1}}{ }_{\mathrm{i}_{2}}, \quad \quad \gamma \in S_{n-2}, \quad k=1 .
$$

For $k \geq 2$, the requisite color factors were constructed by Johansson and Ochirov [44], and so we refer to eq. (2.14) as the Melia-Johansson-Ochirov (MJO) decomposition. ${ }^{6}$ We do not give here the explicit expressions for the JO color factors (which may be found in refs. $[40,44,50])$ but for our five-point example $\mathcal{A}_{5,2}^{\text {bicolor }}$, they reduce to

$$
C_{15342}=c_{1}, \quad C_{13542}=c_{2}+c_{4}, \quad C_{13452}=c_{2}
$$

where $c_{i}$ are defined in eq. (2.7). It is straightforward to verify that the MJO decomposition

$$
\mathcal{A}_{5,2}^{\text {bicolor }}=\tilde{A}^{(\mathrm{s})}(15342) C_{15342}+\tilde{A}^{(\mathrm{s})}(13542) C_{13542}+\tilde{A}^{(\mathrm{s})}(13452) C_{13452}
$$

agrees with eq. (2.6). The general proof was given in ref. [50].

Similarly, we define partial amplitudes $A^{(\mathrm{s})}(\beta)$ with respect to the second group factor $\mathrm{U}(\tilde{N})$,

$$
A^{(\mathrm{s})}(\beta)=\sum_{i} \frac{M_{i, \beta} c_{i}}{d_{i}}
$$

corresponding to the sum over cubic diagrams $i$ whose color factor $\tilde{c}_{i}$ can be drawn in a planar fashion with the external legs in the cyclic order specified by the permutation $\beta$.

\footnotetext{
${ }^{6}$ Recently, a one-loop version of the MJO decomposition has been developed in ref. [54].
} 
Finally, we define double-partial amplitudes corresponding to a sum over diagrams that satisfy both of these criteria simultaneously

$$
m(\alpha \mid \beta)=\sum_{i} \frac{M_{i, \alpha} M_{i, \beta}}{d_{i}} .
$$

In our five-point example, the double-partial amplitudes for which both $\alpha$ and $\beta$ belong to the Melia basis are given by

$$
\begin{gathered}
\left(\begin{array}{cccc}
m(15342 \mid 15342) & m(15342 \mid 13542) & m(15342 \mid 13452) \\
m(13542 \mid 15342) & m(13542 \mid 13542) & m(13542 \mid 13452) \\
m(13452 \mid 15342) & m(13452 \mid 13542) & m(13452 \mid 13452)
\end{array}\right) \\
=\left(\begin{array}{ccc}
\frac{1}{d_{1}}+\frac{1}{d_{3}}+\frac{1}{d_{5}} & -\frac{1}{d_{3}} & -\frac{1}{d_{5}} \\
-\frac{1}{d_{3}} & \frac{1}{d_{3}}+\frac{1}{d_{4}} & -\frac{1}{d_{4}} \\
-\frac{1}{d_{5}} & -\frac{1}{d_{4}} & \frac{1}{d_{2}}+\frac{1}{d_{4}}+\frac{1}{d_{5}}
\end{array}\right) .
\end{gathered}
$$

For purely biadjoint amplitudes [9] and for bicolor amplitudes with a single pair of bifundamentals [48], the double-partial amplitudes are equal to the elements of the propagator matrix defined in ref. [55]. For bicolor amplitudes containing two or more (massless) $\psi \bar{\psi}$ pairs, the matrix of double-partial amplitudes will be a "thinned-out" version of the propagator matrix, because some of the cubic diagrams that contribute to the purely biadjoint amplitude will be absent.

Using eqs. (2.15) and (2.21) we may express the partial amplitudes (2.20) as

$$
A^{(\mathrm{s})}(\beta)=\sum_{\gamma \in \text { Melia }} m(\beta \mid 1 \gamma 2) C_{1 \gamma 2}
$$

and in particular the partial amplitudes belonging to the Melia basis are

$$
A^{(\mathrm{s})}(1 \delta 2)=\sum_{\gamma \in \text { Melia }} m(1 \delta 2 \mid 1 \gamma 2) C_{1 \gamma 2}, \quad \delta \in \text { Melia } .
$$

For amplitudes containing only bifundamental fields $(n=2 k)$, the $(2 k-2) ! / k ! \times(2 k-2) ! / k$ ! matrix whose entries are given by $m(1 \delta 2 \mid 1 \gamma 2)$ may be inverted to give

$$
C_{1 \gamma 2}=\sum_{\delta \in \text { Melia }} m^{-1}(1 \gamma 2 \mid 1 \delta 2) A^{(\mathrm{s})}(1 \delta 2), \quad \text { for } n=2 k .
$$

This may in turn be inserted into eq. (2.14) to yield

$$
\mathcal{A}_{2 k, k}^{\text {bicolor }}=\sum_{\gamma, \delta \in \text { Melia }} \tilde{A}^{(\mathrm{s})}(1 \gamma 2) m^{-1}(1 \gamma 2 \mid 1 \delta 2) A^{(\mathrm{s})}(1 \delta 2)
$$

which has the structure of a KLT-type relation for the bicolor amplitude. The elements of the inverse matrix $m^{-1}(1 \gamma 2 \mid 1 \delta 2)$ are rational functions of the kinematic invariants, but we conjecture that, using momentum conservation, they can be written as polynomials in the kinematic invariants. (We have verified this for $\mathcal{A}_{4,2}^{\text {bicolor }}$ and $\mathcal{A}_{6,3}^{\text {bicolor }}$.) 
When the amplitude contains biadjoint fields as well as bifundamentals $(n>2 k)$, the rank of the matrix $m(1 \delta 2 \mid 1 \gamma 2)$ is less than $(n-2) ! / k !$, and therefore the matrix cannot be inverted. It possesses null vectors: eigenvectors with eigenvalue zero. In the next section, we will use the color-factor symmetry to determine these null vectors. Then in section 4 we will identify an invertible submatrix of this matrix, and thereby write a KLT-type relation for bicolor amplitudes that contain both bifundamental and biadjoint fields.

\section{Color-factor symmetry of the bicolor scalar theory}

A new symmetry of gauge-theory amplitudes was introduced in ref. [39], one which acts on the color factors $c_{i}$ while leaving the amplitude invariant. Specifically, color factors undergo momentum-dependent shifts $\delta c_{i}$ that preserve the Jacobi identities satisfied by the color factors. The proof of invariance of gauge-theory amplitudes under color-factor shifts employed a decomposition called the radiation vertex expansion.

Ref. [40] examined the color-factor symmetry of QCD amplitudes involving $k$ massive quark-antiquark pairs and $n-2 k$ gluons. For each of the external gluon legs $a$ in a QCD amplitude, there is a family of color-factor shifts $\delta_{a} c_{i}$. It was shown that the color factors $C_{1 \gamma 2}$ defined by Johansson and Ochirov transform in a natural way under color-factor shifts

$$
\delta_{a} C_{1 \sigma(3) \cdots \sigma(b-1) a \sigma(b) \cdots \sigma(n) 2}=\alpha_{a, \sigma}\left(k_{a} \cdot k_{1}+\sum_{c=3}^{b-1} k_{a} \cdot k_{\sigma(c)}\right)
$$

where $\alpha_{a, \sigma}$ is a set of arbitrary, independent parameters (or functions) associated with the family of shifts, with $\sigma$ denoting a fixed permutation of the remaining legs $\{3, \cdots, n\} \backslash\{a\}$ that belongs to the Melia basis. To give a specific example, the five-point amplitude $\mathcal{A}_{5,2}^{\text {qcd }}$ is invariant under a one-parameter shift $\delta_{5} c_{i}$ under which the JO color factors transform as

$$
\begin{aligned}
& \delta_{5} C_{15342}=\alpha_{5,34} k_{5} \cdot k_{1}, \\
& \delta_{5} C_{13542}=\alpha_{5,34} k_{5} \cdot\left(k_{1}+k_{3}\right), \\
& \delta_{5} C_{13452}=\alpha_{5,34} k_{5} \cdot\left(k_{1}+k_{3}+k_{4}\right) .
\end{aligned}
$$

In general, since the number of gluons in the amplitude is $n-2 k$ and the number of Melia permutations $\sigma$ is $(n-3) ! / k$ ! the number of independent color-factor shifts is given by $(n-2 k)(n-3) ! / k$ ! for $k \geq 2$. (For $k=0$ and $k=1$, the number of independent shifts is $(n-3)(n-3) !)$

In section 8 of ref. [39] it was shown that amplitudes of the biadjoint scalar theory are also invariant under color-factor shifts, which was proven using the cubic vertex expansion. This proof is straightforwardly extended to the amplitudes of the bicolor scalar theory introduced in the previous section.

The color-factor symmetry of bicolor amplitudes can be used to derive the null eigenvectors of the matrix of double-partial amplitudes. First we use eq. (2.15) and its analog for $\tilde{c}_{i}$ to express the bicolor amplitude (2.5) in terms of the JO color factors

$$
\mathcal{A}_{n, k}^{\text {bicolor }}=\sum_{\gamma, \delta \in \text { Melia }} \tilde{C}_{1 \delta 2} m(1 \delta 2 \mid 1 \gamma 2) C_{1 \gamma 2} .
$$


Its variation under a shift of the color factors $\delta_{a} c_{i}$ is

$$
\delta_{a} \mathcal{A}_{n, k}^{\text {bicolor }}=\sum_{\gamma, \delta \in \text { Melia }} \tilde{C}_{1 \delta 2} m(1 \delta 2 \mid 1 \gamma 2) \delta_{a} C_{1 \gamma 2} .
$$

Since the amplitude is invariant under color-factor shifts, and since $\tilde{C}_{1 \delta 2}$ constitute an independent basis, we conclude that $\delta_{a} C_{1 \gamma 2}$ are null vectors of the matrix $m(1 \delta 2 \mid 1 \gamma 2)$

$$
0=\sum_{\gamma \in \text { Melia }} m(1 \delta 2 \mid 1 \gamma 2) \delta_{a} C_{1 \gamma 2}
$$

Given the independence of $\alpha_{a, \sigma}$ we obtain

$$
\sum_{b=3}^{n+1}\left(k_{a} \cdot k_{1}+\sum_{c=3}^{b-1} k_{a} \cdot k_{\sigma(c)}\right) m(1 \delta 2 \mid 1 \sigma(3) \cdots \sigma(b-1) a \sigma(b) \cdots \sigma(n) 2)=0
$$

i.e., we have derived a set of null eigenvectors of the matrix $m(1 \delta 2 \mid 1 \gamma 2)$. Since the matrix of double-partial amplitudes is symmetric (cf. eq. (2.21)), we can also write

$$
\sum_{b=3}^{n+1}\left(k_{a} \cdot k_{1}+\sum_{c=3}^{b-1} k_{a} \cdot k_{\sigma(c)}\right) m(1 \sigma(3) \cdots \sigma(b-1) a \sigma(b) \cdots \sigma(n) 2 \mid 1 \gamma 2)=0 .
$$

The number of independent null eigenvectors is simply the number of independent colorfactor shifts specified above, namely $(n-2 k)(n-3) ! / k$ ! for $k \geq 2$, and $(n-3)(n-3)$ ! for $k=0$ and $k=1$. Subtracting this from the size of the Melia basis $(n-2) ! / k !$, we obtain the rank of the matrix of double-partial amplitudes [44]

$$
\beta(n, k)= \begin{cases}(n-3) ! & k=0,1,2, \\ (n-3) !(2 k-2) / k ! & k \geq 2 .\end{cases}
$$

The existence of null eigenvectors has two consequences for the partial amplitudes

$$
A^{(\mathrm{s})}(\beta)=\sum_{\gamma \in \text { Melia }} m(\beta \mid 1 \gamma 2) C_{1 \gamma 2} .
$$

First, by virtue of eq. (3.5) they are invariant under the color-factor symmetry

$$
\delta_{a} A^{(\mathrm{s})}(\beta)=\sum_{\gamma \in \text { Melia }} m(\beta \mid 1 \gamma 2) \delta_{a} C_{1 \gamma 2}=0 .
$$

Second, by virtue of eq. (3.7), the set of partial amplitudes $A^{(\mathrm{s})}(1 \gamma 2)$ (where $\gamma$ belongs to the Melia set) are not independent, as they obey the fundamental BCJ relations

$$
\sum_{b=3}^{n+1}\left(k_{a} \cdot k_{1}+\sum_{c=3}^{b-1} k_{a} \cdot k_{\sigma(c)}\right) A^{(\mathrm{s})}(1, \sigma(3), \cdots, \sigma(b-1), a, \sigma(b), \cdots, \sigma(n), 2)=0 .
$$

These relations reduce the number of independent amplitudes to $\beta(n, k)$. 
Using the fundamental BCJ relations (3.11), Johansson and Ochirov identified an independent basis of $\beta(n, k)$ amplitudes for $k \geq 2$, namely those $A^{(\mathrm{s})}(1 \gamma 2)$ for which $\gamma$ belongs to the Melia set and with $\gamma(3)$ restricted to be one of the $\bar{\psi}$ fields, i.e. $\gamma(3)$ belongs to the set $\{3,5,7, \cdots 2 k-1\}$. We refer to this subset of permutations $\gamma$ as the JO set. In the $\mathcal{A}_{5,2}^{\text {bicolor }}\left(\bar{\psi}_{1}, \psi_{2}, \bar{\psi}_{3}, \psi_{4}, \phi_{5}\right)$ example, of the three Melia partial amplitudes $A^{(\mathrm{s})}(15342)$, $A^{(\mathrm{s})}(13542)$, and $A^{(\mathrm{s})}(13452)$, only the last two belong to the JO basis. The JO set of permutations will play a prominent role in the remainder of this paper, in which we use the color-factor symmetry to express color-encoded amplitudes in terms of the JO basis.

For $k=2$ amplitudes, $\gamma(3)$ can only be 3 , so the JO basis consists of the $(n-3)$ ! amplitudes $A(1,3, \sigma, 2)$, in which $\sigma$ is an arbitrary permutation of $\{4, \cdots, n\}$. The same set of $(n-3)$ ! amplitudes provides an independent basis for $k=0$ and $k=1$ amplitudes as well, in which 3 labels one of the biadjoint fields.

\section{KLT-type relations for biadjoint and bicolor amplitudes}

Having set the stage by introducing the bicolor scalar theory and its amplitudes in section 2, and by exploring the consequences of their invariance under color-factor symmetry in section 3, we now derive a KLT-type relation for biadjoint and bicolor amplitudes. The results of this section will facilitate the derivation of KLT-type relations for QCD amplitudes in section 5 .

We first perform a color-factor shift

$$
C_{1 \gamma 2}^{\prime}=C_{1 \gamma 2}+\delta C_{1 \gamma 2}
$$

to set to zero all color factors not belonging to the JO set of permutations $\gamma$. (Color-factor redefinitions of this form were previously discussed in section 5 of ref. [7] for all-gluon amplitudes.) For example, for $\mathcal{A}_{5,2}^{\text {bicolor }}$ we choose $\alpha_{5,34}=-C_{15342} / k_{5} \cdot k_{1}$ in eq. (3.2) to obtain

$$
\begin{aligned}
& C_{15342}^{\prime}=0, \\
& C_{13542}^{\prime}=C_{13542}-\frac{k_{5} \cdot\left(k_{1}+k_{3}\right)}{k_{5} \cdot k_{1}} C_{15342}, \\
& C_{13452}^{\prime}=C_{13452}-\frac{k_{5} \cdot\left(k_{1}+k_{3}+k_{4}\right)}{k_{5} \cdot k_{1}} C_{15342} .
\end{aligned}
$$

In the appendix, we compute the analogous color-factor shift for the five-point biadjoint amplitude $\mathcal{A}_{5,0}^{\text {bicolor }}$ (or the five-gluon amplitude $\mathcal{A}_{5,0}^{\text {qcd }}$ ) from which the procedure for the general $n$-point amplitude will be clear. Since the bicolor amplitude (2.14) is invariant under the color-factor shift (4.1), we may write it as

$$
\mathcal{A}_{n, k}^{\text {bicolor }}=\sum_{\gamma \in \mathrm{JO}} \tilde{A}^{(\mathrm{s})}(1 \gamma 2) C_{1 \gamma 2}^{\prime}
$$

where the sum is now restricted to the JO set of permutations.

An alternative way to obtain the shifted color factors $C_{1 \gamma 2}^{\prime}$ employs the fact that $\tilde{A}^{(\mathrm{s})}(1 \gamma 2)$ with $\gamma \in \mathrm{JO}$ is an independent basis, and that BCJ relations can be used to 


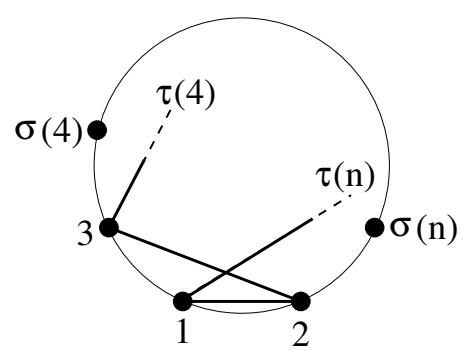

Figure 2. CHY prescription for computing the double-partial amplitude $m(23 \tau 1 \mid 13 \sigma 2)$.

write all the other partial amplitudes $\tilde{A}^{(\mathrm{s})}(1 \gamma 2)$ with $\gamma \in$ Melia in terms of the JO basis, thus reducing eq. (2.14) to the form of eq. (4.3) for some appropriate set of $C_{1 \gamma 2}^{\prime}$. Johansson and Ochirov use this approach to obtain eq. (4.3) with an explicit expression for $C_{1 \gamma 2}^{\prime}$ (cf. eq. (4.45) of ref. [44]).

Since the partial amplitudes (2.23) are invariant under the color-factor shift (cf. eq. (3.10)), we can also write them as a sum over the JO set of permutations

$$
A^{(\mathrm{s})}(\beta)=\sum_{\gamma \in \mathrm{JO}} m(\beta \mid 1 \gamma 2) C_{1 \gamma 2}^{\prime} .
$$

Let us now restrict our attention to amplitudes with two or fewer pairs of bifundamental fields. For $k \leq 2$, permutations belonging to the JO set must have $\gamma(3)=3$, and so eq. (4.4) takes the form

$$
A^{(\mathrm{s})}(\beta)=\sum_{\sigma \in S_{n-3}} m(\beta \mid 13 \sigma 2) C_{13 \sigma 2}^{\prime}, \quad k \leq 2 .
$$

(For $k=0$ and 1, this is true by definition. For $k=2$, this is because 1 and 3 label the only $\bar{\psi}$ fields in the amplitude.) We further restrict our attention to the particular set of partial amplitudes

$$
A^{(\mathrm{s})}(23 \tau 1)=\sum_{\sigma \in S_{n-3}} m(23 \tau 1 \mid 13 \sigma 2) C_{13 \sigma 2}^{\prime}, \quad k \leq 2
$$

where $\tau$ is an arbitrary permutation of $\{4, \cdots, n\}$.

Let us now examine the $(n-3) ! \times(n-3)$ ! matrix of double-partial amplitudes $m(23 \tau 1 \mid 13 \sigma 2)$ for amplitudes with $k \leq 2$, beginning with $k=0$. For the biadjoint scalar theory, Cachazo, He, and Yuan gave an algorithm [9] for computing double-partial amplitudes that begins with drawing a circle with the labels $\{1,3, \sigma(4), \cdots, \sigma(n), 2\}$ around the perimeter, and then inscribing a polygon whose vertices are $\{2,3, \tau(4), \cdots, \tau(n), 1\}$ inside, as shown in figure 2. Because the segments of the polygon emerging from 1 and 2 must cross, the CHY prescription dictates that $m(23 \tau 1 \mid 13 \sigma 2)$ is given by $1 / s_{12}$ times an $(n-1)$-point double-partial amplitude $m(v 3 \tau \mid v 3 \sigma)$, in which one of the fields $(v)$ is off-shell. Since $k_{v}$ can be eliminated in terms of $k_{3}, \cdots, k_{n}$ using momentum conservation, the double-partial amplitudes $m(v 3 \tau \mid v 3 \sigma)$ can be expressed in terms of invariants $s_{a b}$ with $3 \leq a, b \leq n$. For example, for the amplitude $\mathcal{A}_{5,0}^{\text {bicolor }}$, one has

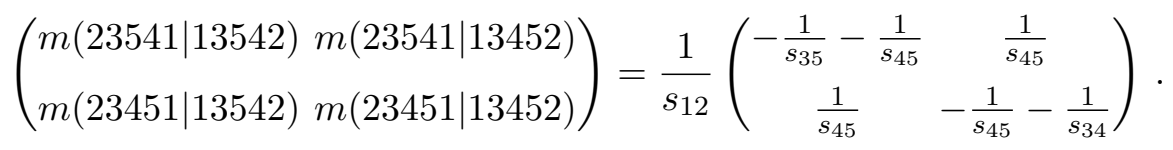


The inverse of this matrix (using momentum conservation) is given by

$$
\left(\begin{array}{cc}
-s_{35}\left(s_{34}+s_{45}\right) & -s_{34} s_{35} \\
-s_{34} s_{35} & -s_{34}\left(s_{35}+s_{45}\right)
\end{array}\right)=-\left(\begin{array}{cc}
S[54 \mid 54]_{3} & S[54 \mid 45]_{3} \\
S[45 \mid 54]_{3} & S[45 \mid 45]_{3}
\end{array}\right)
$$

where $S[\sigma \mid \tau]_{3}$ is the momentum kernel $[4-7]$ defined as $^{7}$

$$
S[\sigma \mid \tau]_{3}=\prod_{i=4}^{n}\left[s_{3, \sigma(i)}+\sum_{j=4}^{i-1} \theta(\sigma(j), \sigma(i))_{\tau} s_{\sigma(j), \sigma(i)}\right] .
$$

For $n$-point amplitudes in general, the inverse of $m(23 \tau 1 \mid 13 \sigma 2)$ is given by the negative of the momentum kernel, $-S[\sigma \mid \tau]_{3}$, as was shown by Cachazo, He, and Yuan [9] by using KLT orthogonality [56]. This can be seen from the results of ref. [9] by relabeling the external legs $1 \rightarrow 3, n-1 \rightarrow 1, n \rightarrow 2$, and $\sigma(i) \rightarrow \sigma(i+2)$, and then using cyclic symmetry of the double-partial amplitudes $m(3 \tau 12 \mid 3 \sigma 21)=m(23 \tau 1 \mid 13 \sigma 2)$.

Next consider bicolor amplitudes with one pair of (possibly massive) bifundamentals, $\bar{\psi}_{1}$ and $\psi_{2}$. It was shown in ref. [48] that the double-partial amplitudes with $k=1$, when expressed in terms of $k_{a} \cdot k_{b}$ with $2 \leq a, b \leq n$, are identical to those of the biadjoint theory. Above we showed that the particular double-partial amplitudes $m(23 \tau 1 \mid 13 \sigma 2)$ can be written ${ }^{8}$ in terms of $k_{a} \cdot k_{b}$ with $3 \leq a, b \leq n$. Since $s_{a b}=2 k_{a} \cdot k_{b}$ for $3 \leq a, b \leq n$, the matrix of double-partial amplitudes $m(23 \tau 1 \mid 13 \sigma 2)$ with $k=1$ is identical, when written in terms of $s_{a b}$ with $3 \leq a, b \leq n$, to that of the biadjoint scalar theory, and therefore has the same inverse, namely $-S[\sigma \mid \tau]_{3}$.

Next let us consider bicolor amplitudes with two pairs of bifundamentals, $\bar{\psi}_{1}, \psi_{2}$ and $\bar{\psi}_{3}, \psi_{4}$, with masses $m_{1}=m_{2}$ and $m_{3}=m_{4}$. In general, fewer cubic diagrams will contribute to these double-partial amplitudes relative to purely biadjoint double-partial amplitudes because some of the cubic vertices present in the latter are ruled out by flavor conservation, etc. (e.g., $\bar{\psi}_{1} \bar{\psi}_{3} \phi_{a}$ and $\bar{\psi}_{1} \psi_{4} \phi_{a}$ ). For the specific subclass of double-partial amplitudes $m(23 \tau 1 \mid 13 \sigma 2)$, however, the same set of cubic diagrams that contribute in the purely biadjoint case will also contribute to $k=2$ bicolor amplitudes. This is because for these amplitudes, as we explained above, the fields $\bar{\psi}_{1}, \psi_{2}$ are effectively replaced by a virtual biadjoint field $\phi_{v}$, and the $n$-point double-partial amplitude is given by $1 / s_{12}$ times an $(n-1)$-point double-partial amplitude with $(n-3)$ external biadjoint fields, one virtual biadjoint field, and one bifundamental pair $\bar{\psi}_{3}, \psi_{4}$. These latter (effectively $k=1$ ) double-partial amplitudes are equal to the analogous biadjoint double-partial amplitudes, except that we must replace $s_{i j}$ with $s_{i j}^{\prime}$, where

$$
\begin{array}{llrl}
s_{12}^{\prime} & =s_{12}, & s_{34}^{\prime}=s_{34}, & s_{a b}^{\prime}=s_{a b}, \\
s_{3 a}^{\prime}=s_{3 a}-m_{3}^{2}, & s_{4 a}^{\prime}=s_{4 a}-m_{3}^{2}, & a, b \geq 5 .
\end{array}
$$

\footnotetext{
${ }^{7}$ Here $\sigma, \tau \in S_{n-3}$ are permutations acting on labels $\{4, \cdots, n\}$. Define $\theta(r, s)_{\tau}=1$ if the ordering of $r, s$ is the same in both sequences of labels, $\{\sigma(4), \cdots, \sigma(n)\}$ and $\{\tau(4), \cdots, \tau(n)\}$, and zero otherwise. The original definition [4-7] of the momentum kernel is slightly modified [8,9] to be symmetric in its arguments, $S[\sigma \mid \tau]_{3}=S[\tau \mid \sigma]_{3}$.

${ }^{8} \mathrm{U} \operatorname{sing} s_{12}=\left(k_{3}+\cdots k_{n}\right)^{2}$.
} 
For example, for $\mathcal{A}_{5,2}^{\text {bicolor }}$, eq. (4.7) becomes

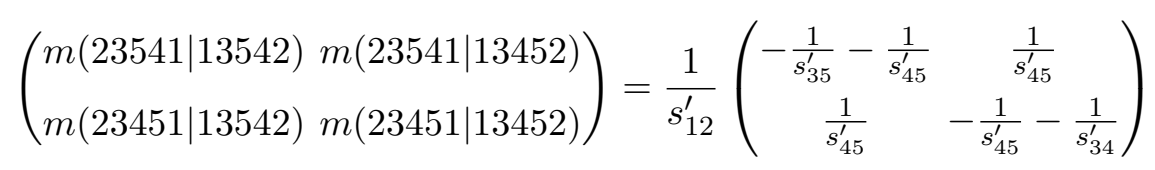

as can be verified by examining eqs. (2.7) and (2.22). In general, for $k=2$ amplitudes, the inverse of $m(23 \tau 1 \mid 13 \sigma 2)$ is given by (minus) the momentum kernel (4.9) with $s_{i j} \rightarrow s_{i j}^{\prime}$.

Thus, for all bicolor amplitudes with $k \leq 2$, we have established that the inverse of the matrix of double-partial amplitudes $m(23 \tau 1 \mid 13 \sigma 2)$ is given by (minus) the momentum kernel, $-S[\sigma \mid \tau]_{3}$ (with $s_{i j} \rightarrow s_{i j}^{\prime}$ in the $k=2$ case). Hence eq. (4.6) can be inverted to give an explicit expression for the shifted color factors

$$
C_{13 \sigma 2}^{\prime}=-\sum_{\tau \in S_{n-3}} S[\sigma \mid \tau]_{3} A^{(\mathrm{s})}(23 \tau 1), \quad k \leq 2 .
$$

In turn, eq. (4.12) can be inserted into eq. (4.3) to obtain the following expression for $k \leq 2$ bicolor amplitudes ${ }^{9}$

$$
\mathcal{A}_{n, k \leq 2}^{\text {bicolor }}=-\sum_{\sigma, \tau \in S_{n-3}} \tilde{A}^{(\mathrm{s})}(13 \sigma 2) S[\sigma \mid \tau]_{3} A^{(\mathrm{s})}(23 \tau 1) .
$$

This is our new KLT-type relation for biadjoint and bicolor scalar amplitudes (with $k \leq 2$ ), which expresses the full amplitude in terms of dual partial scalar amplitudes $A^{(\mathrm{s})}(\cdots)$ and $\tilde{A}^{(\mathrm{s})}(\cdots)$. For example, for the five-point amplitude we have

$$
\mathcal{A}_{5,2}^{\text {bicolor }}=\left(\tilde{A}^{(\mathrm{s})}(13542), \tilde{A}^{(\mathrm{s})}(13452)\right)\left(\begin{array}{cc}
-s_{35}^{\prime}\left(s_{34}^{\prime}+s_{45}^{\prime}\right) & -s_{34}^{\prime} s_{35}^{\prime} \\
-s_{34}^{\prime} s_{35}^{\prime} & -s_{34}^{\prime}\left(s_{35}^{\prime}+s_{45}^{\prime}\right)
\end{array}\right)\left(\begin{array}{c}
A^{(\mathrm{s})}(23541) \\
A^{(\mathrm{s})}(23451)
\end{array}\right) .
$$

We can also write an expression for the most general partial scalar amplitude $A^{(\mathrm{s})}(\beta)$ in terms of the partial amplitudes belonging to the independent basis $A^{(\mathrm{s})}(23 \tau 1)$,

$$
A^{(\mathrm{s})}(\beta)=-\sum_{\sigma, \tau \in S_{n-3}} m(\beta \mid 13 \sigma 2) S[\sigma \mid \tau]_{3} A^{(\mathrm{s})}(23 \tau 1), \quad k \leq 2
$$

by inserting eq. (4.12) into eq. (4.4). These are precisely BCJ relations for scalar partial amplitudes.

For amplitudes with $k>2$, the fundamental BCJ relations imply that the matrix of double-partial amplitudes has rank $\beta(n, k)$, defined in eq. (3.8). In general, the $\beta(n, k) \times \beta(n, k)$ submatrix $m(2 \delta 1 \mid 1 \gamma 2)$, where both $\delta$ and $\gamma$ belong to the JO set, should be invertible. Provided this is the case, the equation ${ }^{10}$

$$
A^{(\mathrm{s})}(2 \delta 1)=\sum_{\gamma \in \mathrm{JO}} m(2 \delta 1 \mid 1 \gamma 2) C_{1 \gamma 2}^{\prime}, \quad \delta \in \mathrm{JO}
$$

\footnotetext{
${ }^{9}$ Recall that for $k=2$ we must let $s_{i j} \rightarrow s_{i j}^{\prime}$ in eq. (4.9).

${ }^{10}$ The partial amplitudes $A^{(\mathrm{s})}(2 \delta 1)$ constitute an alternative JO basis with opposite choice of "signature" [43] for the $\bar{\psi}_{1}, \psi_{2}$ bifundamental pair relative to the usual JO basis.
} 
can be inverted to give

$$
C_{1 \gamma 2}^{\prime}=\sum_{\delta \in \mathrm{JO}} T(1 \gamma 2 \mid 2 \delta 1) A^{(\mathrm{s})}(2 \delta 1), \quad \gamma \in \mathrm{JO}
$$

although, unlike the $k \leq 2$ case, we cannot present at this point an explicit expression for the inverse matrix $T$. Inserting eq. (4.17) into eq. (4.3), we can write a KLT-type relation for a general bicolor amplitude

$$
\mathcal{A}_{n, k}^{\text {bicolor }}=\sum_{\gamma, \delta \in \mathrm{JO}} \tilde{A}^{(\mathrm{s})}(1 \gamma 2) T(1 \gamma 2 \mid 2 \delta 1) A^{(\mathrm{s})}(2 \delta 1) .
$$

Similarly, by inserting eq. (4.17) into eq. (4.4), we obtain the BCJ relations

$$
A^{(\mathrm{s})}(\beta)=\sum_{\gamma, \delta \in \mathrm{JO}} m(\beta \mid 1 \gamma 2) T(1 \gamma 2 \mid 2 \delta 1) A^{(\mathrm{s})}(2 \delta 1) .
$$

We anticipate that, like the momentum kernel, the matrix elements of $T(1 \gamma 2 \mid 2 \delta 1)$ will be $(n-3)^{\text {th }}$ order polynomials in the kinematic invariants.

\section{KLT-type relations for QCD (and gravity) amplitudes}

In this section, we derive KLT-type relations for QCD amplitudes with quarks and gluons in two different ways. The first way uses the invariance of QCD amplitudes under colorfactor symmetry $[39,40]$, together with the results of the previous section. The second way explicitly invokes color-kinematic duality [10, 44]. Both methods of course yield the same result. In the final subsection, we apply our methods to obtain KLT relations for gravitational amplitudes.

\subsection{Derivation of QCD KLT-type relations using color-factor symmetry}

An $n$-point tree-level QCD amplitude with $k$ differently flavored (massive) quarks $\psi, k$ (massive) anti-quarks $\bar{\psi}$ with corresponding anti-flavors, and $(n-2 k)$ gluons is given by a sum over cubic diagrams

$$
\mathcal{A}_{n, k}^{\mathrm{qcd}}\left(\bar{\psi}_{1}, \psi_{2}, \bar{\psi}_{3}, \psi_{4}, \cdots, \bar{\psi}_{2 k-1}, \psi_{2 k}, g_{2 k+1}, \cdots, g_{n}\right)=\sum_{i \in \text { cubic }} \frac{c_{i} n_{i}}{d_{i}}
$$

where $c_{i}$ and $d_{i}$ are the same color factors and propagators appearing in section 2 and $n_{i}$ are kinematic numerators for QCD. Using eq. (2.15), we can rewrite eq. (5.1) in the Melia-Johansson-Ochirov proper decomposition [42-44, 50]

$$
\mathcal{A}_{n, k}^{\mathrm{qcd}}=\sum_{\gamma \in \text { Melia }} A(1 \gamma 2) C_{1 \gamma 2}
$$

where $C_{1 \gamma 2}$ are the JO color factors described in section 2 and

$$
A(\alpha)=\sum_{i} \frac{M_{i, \alpha} n_{i}}{d_{i}}
$$

are color-ordered partial amplitudes of the gauge theory. 
For amplitudes containing one or more gluons, $\mathcal{A}_{n, k}^{\mathrm{qcd}}$ is invariant under a family of color-factor shifts, as was shown in ref. [39] by expanding the amplitude in a radiation vertex expansion. Invariance of eq. (5.2) under a color-factor shift $\delta_{a} c_{i}$ implies

$$
\delta_{a} \mathcal{A}_{n, k}^{\mathrm{qcd}}=\sum_{\gamma \in \text { Melia }} A(1 \gamma 2) \delta_{a} C_{1 \gamma 2}=0
$$

where $\delta_{a} C_{1 \gamma 2}$ are given in eq. (3.1). Since the parameters $\alpha_{a, \sigma}$ in eq. (3.1) are independent, eq. (5.4) implies

$$
\sum_{b=3}^{n+1}\left(k_{a} \cdot k_{1}+\sum_{c=3}^{b-1} k_{a} \cdot k_{\sigma(c)}\right) A(1, \sigma(3), \cdots, \sigma(b-1), a, \sigma(b), \cdots, \sigma(n), 2)=0
$$

which are simply the fundamental BCJ relations for color-ordered QCD amplitudes, derived using color-factor symmetry. These relations were first discovered for all-gluon amplitudes in ref. [10], and proven in refs. [57-60]. The fundamental BCJ relations were extended to QCD amplitudes for all values of $k$ in ref. [44], and subsequently proven in ref. [61]. They were shown to be a consequence of color-factor symmetry in refs. [39, 40].

Using the color-factor symmetry of the amplitude, we can rewrite eq. (5.2) in terms of shifted color factors $C_{1 \gamma 2}^{\prime}$ defined in section 4 , which vanish unless $\gamma$ belongs to the JO set of permutations

$$
\mathcal{A}_{n, k}^{\mathrm{qcd}}=\sum_{\gamma \in \mathrm{JO}} A(1 \gamma 2) C_{1 \gamma 2}^{\prime} .
$$

For amplitudes with $k \leq 2$, we can use eq. (4.12) to write ${ }^{11}$

$$
\mathcal{A}_{n, k \leq 2}^{\mathrm{qcd}}=-\sum_{\sigma, \tau \in S_{n-3}} A(13 \sigma 2) S[\sigma \mid \tau]_{3} A^{(\mathrm{s})}(23 \tau 1) .
$$

This is our KLT-type expression for QCD amplitudes with two or fewer quark-antiquark pairs. For $k=0$, an expression equivalent to this first appeared in ref. [33] and was proven in ref. [34] using BCFW techniques [35]. In this paper, we have established that eq. (5.7) is also valid for amplitudes with $k=1$ and $k=2$. We emphasize that each of the terms in the sum is both gauge-invariant (the partial gauge-theory amplitudes) and color-factor symmetric (the dual partial scalar amplitudes), as is, of course, the entire color-encoded amplitude.

For amplitudes with more than two quark-antiquark pairs, we use eq. (4.17) to obtain

$$
\mathcal{A}_{n, k}^{\mathrm{qcd}}=\sum_{\gamma, \delta \in \mathrm{JO}} A(1 \gamma 2) T(1 \gamma 2 \mid 2 \delta 1) A^{(\mathrm{s})}(2 \delta 1)
$$

where $T(1 \gamma 2 \mid 2 \delta 1)$ is the inverse of the matrix $m(2 \delta 1 \mid 1 \gamma 2)$, for both $\delta$ and $\gamma$ belonging to the JO set. For all-quark amplitudes $(n=2 k)$, there is no color-factor symmetry, and eq. (2.25) may be used to rewrite eq. (5.2) as

$$
\mathcal{A}_{2 k, k}^{\mathrm{qcd}}=\sum_{\gamma, \delta \in \text { Melia }} A(1 \gamma 2) m^{-1}(1 \gamma 2 \mid 1 \delta 2) A^{(\mathrm{s})}(1 \delta 2) .
$$

We cannot present at this point explicit expressions for $T$ or $m^{-1}$ in these two equations, but we anticipate that they take the form of $(n-3)^{\text {th }}$ order polynomials of kinematic invariants.

\footnotetext{
${ }^{11}$ For $k=2$, one must let $s_{i j} \rightarrow s_{i j}^{\prime}$ in eq. (4.9), where $s_{i j}^{\prime}$ are defined in eq. (4.10).
} 


\subsection{Derivation of QCD KLT-type relations using generalized gauge invariance}

An alternative proof of the KLT-type relations for QCD amplitudes begins by assuming color-kinematic duality, which means that the kinematic numerators satisfy the same algebraic relations as the color factors $[10,44]$. The Jacobi relations among the color factors $c_{i}$ allow them to be written in terms of a set of independent color factors $C_{1 \gamma 2}$ as in eq. (2.15). The analogous kinematic Jacobi equations allow the kinematic numerators $n_{i}$ to be written as

$$
n_{i}=\sum_{\gamma \in \text { Melia }} M_{i, 1 \gamma 2} N_{1 \gamma 2}
$$

for some set of independent numerators $N_{1 \gamma 2}$. Expressions for $N_{1 \gamma 2}$ in terms of $n_{i}$ parallel those for $C_{1 \gamma 2}$ in terms of $c_{i}$ [44]. We use eq. (5.10), together with eq. (2.20), to write eq. (5.1) as

$$
\mathcal{A}_{n, k}^{\mathrm{qcd}}=\sum_{\gamma \in \text { Melia }} A^{(\mathrm{s})}(1 \gamma 2) N_{1 \gamma 2}
$$

as was done for all-gluon amplitudes $(k=0)$ in ref. [11]. Similarly, the color-ordered partial amplitudes (5.3) can be written as

$$
A(\alpha)=\sum_{\gamma \in \text { Melia }} m(\alpha \mid 1 \gamma 2) N_{1 \gamma 2}
$$

using eq. (2.21).

Kinematic numerators $n_{i}$ that satisfy the same algebraic relations as color factors $c_{i}$ can necessarily be written in the form (5.10), but this requirement does not uniquely determine the $N_{1 \gamma 2}$ (unless there are no gluons, $n=2 k$ ). Generalized gauge transformations on $n_{i}$ (provided they preserve the Jacobi relations) can result in a different set of $N_{1 \gamma 2}$ 's. In ref. [39], we described a set of restricted generalized gauge transformations of all-gluon amplitudes that preserve the Jacobi relations, analogous to the shifts of color factors. In the context of QCD amplitudes of gluons and quarks, for each of the external gluon legs $a$, there is a family of restricted generalized gauge transformations $\tilde{\delta}_{a} n_{i}$. These restricted generalized gauge transformations act on the independent numerators $N_{1 \gamma 2}$ as

$$
\tilde{\delta}_{a} N_{1 \sigma(3) \cdots \sigma(b-1) a \sigma(b) \cdots \sigma(n) 2}=\beta_{a, \sigma}\left(k_{a} \cdot k_{1}+\sum_{c=3}^{b-1} k_{a} \cdot k_{\sigma(c)}\right)
$$

where $\beta_{a, \sigma}$ is a set of arbitrary, independent parameters (or functions), with $\sigma$ denoting a fixed permutation of the remaining legs $\{3, \cdots, n\} \backslash\{a\}$ that belongs to the Melia basis. We can choose $\beta_{a, \sigma}$ in such a way that the shifted numerators

$$
N_{1 \gamma 2}^{\prime}=N_{1 \gamma 2}+\tilde{\delta} N_{1 \gamma 2}
$$

vanish except for those in which $\gamma$ is restricted to the JO set. We described how to do this for color factors in section 4 and in the appendix, and the procedure is the same in this case. Since gauge-theory amplitudes are invariant under generalized gauge transformations, 
eqs. (5.11) and (5.12) can be written as

$$
\begin{aligned}
& \mathcal{A}_{n, k}^{\mathrm{qcd}}=\sum_{\gamma \in \mathrm{JO}} A^{(\mathrm{s})}(1 \gamma 2) N_{1 \gamma 2}^{\prime}, \\
& A(\alpha)=\sum_{\gamma \in \mathrm{JO}} m(\alpha \mid 1 \gamma 2) N_{1 \gamma 2}^{\prime}
\end{aligned}
$$

where the sums are now restricted to the JO set of permutations.

For amplitudes with two or fewer quark-antiquark pairs, we may invert eq. (5.16), just as we did in section 4 , to obtain

$$
N_{13 \sigma 2}^{\prime}=-\sum_{\tau \in S_{n-3}} S[\sigma \mid \tau]_{3} A(23 \tau 1), \quad k \leq 2 .
$$

For all-gluon amplitudes $(k=0)$, these numerators are essentially those written down by Kiermaier in ref. [62], and later by Cachazo, He, and Yuan in ref. [9].

By inserting eq. (5.17) into eq. (5.15), we obtain the KLT-type relation

$$
\mathcal{A}_{n, k \leq 2}^{\mathrm{qcd}}=-\sum_{\sigma, \tau \in S_{n-3}} A^{(\mathrm{s})}(13 \sigma 2) S[\sigma \mid \tau]_{3} A(23 \tau 1),
$$

which is equivalent to eq. (5.7). Similarly, for $k>2$, we can obtain an expression equivalent to eq. (5.8).

By inserting eq. (5.17) into eq. (5.16), we obtain an expression for the most general color-ordered amplitude $A(\alpha)$ in terms of partial amplitudes belonging to the independent basis $A(23 \tau 1)$, namely

$$
A(\alpha)=-\sum_{\sigma, \tau \in S_{n-3}} m(\alpha \mid 13 \sigma 2) S[\sigma \mid \tau]_{3} A(23 \tau 1), \quad k \leq 2 .
$$

In the all-gluon case $(k=0)$, this is essentially the expression given in appendix $\mathrm{C}$ of ref. [63]. It is equivalent in content (for $k \leq 2$ ) to eq. (4.37) of ref. [44], though different in form.

\subsection{Gravitational KLT amplitudes}

Having invoked in the previous subsection color-kinematic duality for the QCD kinematic numerators $n_{i}$, we can now use the double-copy prescription $[10,11,17]$ to obtain gravitational scattering amplitudes containing $n-2 k$ gravitons and $2 k$ matter particles $[44,64,65]$

$$
\mathcal{A}_{n, k}^{\text {grav }}=\sum_{i \in \text { cubic }} \frac{n_{i} \tilde{n}_{i}}{d_{i}} .
$$

Color-kinematic duality then allows us to use eq. (5.10) to write

$$
\mathcal{A}_{n, k}^{\text {grav }}=\sum_{\gamma \in \text { Melia }} \tilde{A}(1 \gamma 2) N_{1 \gamma 2}
$$

where $\tilde{A}(1 \gamma 2)$ are QCD partial amplitudes (5.3) with $n_{i}$ replaced by $\tilde{n}_{i}$. This expression was first introduced for all-graviton amplitudes $(k=0)$ in ref. [11]. By virtue of the fact 
that the QCD partial amplitudes $\tilde{A}(1 \gamma 2)$ obey BCJ relations (5.5), the amplitude (5.21) is invariant under (restricted) generalized gauge transformations

$$
\tilde{\delta} \mathcal{A}_{n, k}^{\text {grav }}=\sum_{\gamma \in \text { Melia }} \tilde{A}(1 \gamma 2) \tilde{\delta} N_{1 \gamma 2}=0 .
$$

Hence we can write eq. (5.21) in terms of shifted numerators (5.14)

$$
\mathcal{A}_{n, k}^{\text {grav }}=\sum_{\gamma \in \mathrm{JO}} \tilde{A}(1 \gamma 2) N_{1 \gamma 2}^{\prime}
$$

with the sum now restricted to the JO set of permutations. We may then use eq. (5.17) we obtain the gravitational KLT relation ${ }^{12}$

$$
\mathcal{A}_{n, k \leq 2}^{\text {grav }}=-\sum_{\sigma, \tau \in S_{n-3}} \tilde{A}(13 \sigma 2) S[\sigma \mid \tau]_{3} A(23 \tau 1)
$$

valid $^{13}$ for amplitudes with $k \leq 2$. A similar expression can be written for amplitudes with $k>2$. For $k=0$, this is just the original (field-theory) KLT relation for the tree-level $n$-graviton amplitude, so we have come full circle to the starting point of this paper.

\section{Conclusions}

Gauge-theory amplitudes possess a color-factor symmetry, which acts on its color factors $c_{i}$ via momentum-dependent shifts while leaving the tree-level amplitudes invariant. A direct consequence of this symmetry are the fundamental BCJ relations satisfied by the color-ordered partial amplitudes of Yang-Mills theory and QCD.

The biadjoint scalar theory, which can be considered the zeroth copy of Yang-Mills theory, also possesses color-factor symmetry. In this paper, we introduced another theory with color-factor symmetry, the bicolor scalar theory. This theory contains both massless biadjoint scalars as well as massive bifundamental scalars, and can be regarded as the zeroth copy of QCD. The partial amplitudes of the biadjoint and bicolor scalar theories are dual to the partial amplitudes of Yang-Mills and QCD, as they can be obtained by replacing the kinematic numerators $n_{i}$ in the latter with color factors $\tilde{c}_{i}$. We showed that the dual partial amplitudes are themselves invariant under color-factor symmetry, and also obey BCJ relations.

The color-factor symmetry was then used to recast tree-level biadjoint and bicolor amplitudes into a KLT-type form, involving a sum over products of dual partial amplitudes multiplied by a momentum-dependent function $T(\cdots)$. This momentum-dependent function is given by the inverse of a particular submatrix of double-partial amplitudes of the bicolor theory, and is thus a rational function of the kinematic invariants. For amplitudes with two or fewer bifundamental pairs $(k \leq 2)$, this function was shown to be an $(n-3)^{\text {th }}$

\footnotetext{
${ }^{12}$ Earlier work on extensions of KLT relations to more general gravitational amplitudes includes refs. [33, 45-47].

${ }^{13}$ Recall that for $k=2$ we must let $s_{i j} \rightarrow s_{i j}^{\prime}$ in eq. (4.9).
} 
degree polynomial of the kinematic invariants, specifically the momentum kernel of refs. [49], slightly modified by masses in the $k=2$ case. We conjecture that, for amplitudes with $k>2$, the momentum-dependent function will also be an $(n-3)^{\text {th }}$ degree polynomial in the invariants, for which we hope that an explicit expression can be found.

We also used the color-factor symmetry to obtain a new KLT-type relation for treelevel QCD amplitudes, involving a sum over products of QCD partial amplitudes and dual partial amplitudes, and the same momentum-dependent function that appeared in the bicolor amplitude. Each term in this sum is both gauge-invariant and color-factor symmetric, as is the full color-ordered amplitude. The KLT-type relation was then alternatively obtained through a derivation that explicitly invoked color-kinematic duality and a restricted generalized gauge transformation. Finally, the double-copy prescription together with the same generalized gauge transformation was used to obtain the KLT relation for gravitational amplitudes.

By utilizing the color-factor symmetry and its parallels with generalized gauge transformations, we have presented a unified treatment of the derivation of KLT-type relations for the tree-level amplitudes

$$
\begin{aligned}
\mathcal{A}_{n, k}^{\text {bicolor }} & =\sum_{\gamma, \delta \in \mathrm{JO}} \tilde{A}^{(\mathrm{s})}(1 \gamma 2) T(1 \gamma 2 \mid 2 \delta 1) A^{(\mathrm{s})}(2 \delta 1), \\
\mathcal{A}_{n, k}^{\mathrm{qcd}} & =\sum_{\gamma, \delta \in \mathrm{JO}} A(1 \gamma 2) T(1 \gamma 2 \mid 2 \delta 1) A^{(\mathrm{s})}(2 \delta 1), \\
\mathcal{A}_{n, k}^{\text {grav }} & =\sum_{\gamma, \delta \in \mathrm{JO}} \tilde{A}(1 \gamma 2) T(1 \gamma 2 \mid 2 \delta 1) A(2 \delta 1)
\end{aligned}
$$

of the bicolor scalar theory, QCD, and gravity.

\section{Acknowledgments}

This material is based upon work supported by the National Science Foundation under Grants Nos. PHY17-20202 and PFI:BIC 1318206. RWB is also supported by funds made available through a CWRU Institute Professorship Chair.

\section{A Color-factor shifts for five- and higher-point amplitudes}

In this appendix, we describe the specific color-factor shift required to write the five-gluon amplitude (or five-point biadjoint amplitude) in terms of BCJ basis amplitudes. In the process, we obtain the five-point BCJ relations [10]. Our procedure readily generalizes to an arbitrary $n$-point amplitude.

The Del Duca-Dixon-Maltoni decomposition [51] of the five-gluon amplitude (or alternatively the five-point amplitude of the biadjoint scalar theory) is given by

$$
\begin{aligned}
\mathcal{A}_{5,0}= & \sum_{\gamma \in S_{3}} A(1 \gamma 2) C_{1 \gamma 2} \\
= & A(13452) C_{13452}+A(13542) C_{13542}+A(14352) C_{14352} \\
& +A(15342) C_{15342}+A(14532) C_{14532}+A(15432) C_{15432}
\end{aligned}
$$


where $A(1 \gamma 2)$ constitute the Kleiss-Kuijf basis of partial amplitudes of the corresponding theory. There is a four-parameter family of color-factor shifts generated by gluons 4 and 5

$$
\begin{aligned}
& \delta C_{13452}=\alpha_{4,35} k_{4} \cdot\left(k_{1}+k_{3}\right)+\alpha_{5,34} k_{5} \cdot\left(k_{1}+k_{3}+k_{4}\right), \\
& \delta C_{13542}=\alpha_{4,35} k_{4} \cdot\left(k_{1}+k_{3}+k_{5}\right)+\alpha_{5,34} k_{5} \cdot\left(k_{1}+k_{3}\right), \\
& \delta C_{14352}=\alpha_{4,35} k_{4} \cdot k_{1}+\alpha_{5,43} k_{5} \cdot\left(k_{1}+k_{3}+k_{4}\right), \\
& \delta C_{15342}=\alpha_{4,53} k_{4} \cdot\left(k_{1}+k_{3}+k_{5}\right)+\alpha_{5,34} k_{5} \cdot k_{1}, \\
& \delta C_{14532}=\alpha_{4,53} k_{4} \cdot k_{1}+\alpha_{5,43} k_{5} \cdot\left(k_{1}+k_{4}\right), \\
& \delta C_{15432}=\alpha_{4,53} k_{4} \cdot\left(k_{1}+k_{5}\right)+\alpha_{5,43} k_{5} \cdot k_{1} .
\end{aligned}
$$

We now show explicitly how to define a set of shifted color factors

$$
C_{1 \gamma 2}^{\prime}=C_{1 \gamma 2}+\delta C_{1 \gamma 2}
$$

that vanish except for $C_{13452}^{\prime}$ and $C_{13542}^{\prime}$. First we consider the color factors in which the label 3 is to the right of both 4 and 5 . We set $\delta C_{14532}=-C_{14532}$ and $\delta C_{15432}=-C_{15432}$ by requiring

$$
\left(\begin{array}{cc}
k_{4} \cdot k_{1} & k_{5} \cdot\left(k_{1}+k_{4}\right) \\
k_{4} \cdot\left(k_{1}+k_{5}\right) & k_{5} \cdot k_{1}
\end{array}\right)\left(\begin{array}{l}
\alpha_{4,53} \\
\alpha_{5,43}
\end{array}\right)=-\left(\begin{array}{l}
C_{14532} \\
C_{15432}
\end{array}\right)
$$

which can be solved to give

$$
\left(\begin{array}{l}
\alpha_{4,53} \\
\alpha_{5,43}
\end{array}\right)=\frac{1}{\left(k_{4} \cdot k_{5}\right)\left(k_{2} \cdot k_{3}\right)}\left(\begin{array}{cc}
k_{5} \cdot k_{1} & -k_{5} \cdot\left(k_{1}+k_{4}\right) \\
-k_{4} \cdot\left(k_{1}+k_{5}\right) & k_{4} \cdot k_{1}
\end{array}\right)\left(\begin{array}{l}
C_{14532} \\
C_{15432}
\end{array}\right) .
$$

Next we set $\delta C_{14352}=-C_{14352}$ and $\delta C_{15342}=-C_{15342}$ by choosing

$$
\begin{aligned}
& \alpha_{4,35}=-\frac{C_{14352}}{k_{4} \cdot k_{1}}-\frac{k_{5} \cdot\left(k_{1}+k_{3}+k_{4}\right)}{k_{4} \cdot k_{1}} \alpha_{5,43}, \\
& \alpha_{5,34}=-\frac{C_{15342}}{k_{5} \cdot k_{1}}-\frac{k_{4} \cdot\left(k_{1}+k_{3}+k_{5}\right)}{k_{5} \cdot k_{1}} \alpha_{4,53} .
\end{aligned}
$$

Finally we plug eqs. (A.5) and (A.6) into the first two equations of eq. (A.2) to obtain, after the use of momentum conservation and some algebra,

$$
\begin{aligned}
& C_{13452}^{\prime}=C_{13452}- \frac{k_{4} \cdot\left(k_{1}+k_{3}\right)}{k_{4} \cdot k_{1}} C_{14352}+\frac{k_{5} \cdot k_{2}}{k_{5} \cdot k_{1}} C_{15342} \\
& \quad-\frac{k_{2} \cdot k_{5}}{k_{2} \cdot k_{3}}\left(\frac{k_{4} \cdot k_{3}}{k_{4} \cdot k_{1}}\right) C_{14532}+\frac{k_{2} \cdot k_{5}}{k_{2} \cdot k_{3}}\left(\frac{k_{4} \cdot k_{2}}{k_{5} \cdot k_{1}}-1\right) C_{15432}, \\
& C_{13542}^{\prime}=C_{13542}+ \frac{k_{4} \cdot k_{2}}{k_{4} \cdot k_{1}} C_{14352}-\frac{k_{5} \cdot\left(k_{1}+k_{3}\right)}{k_{5} \cdot k_{1}} C_{15342} \\
&+\frac{k_{4} \cdot k_{2}}{k_{2} \cdot k_{3}}\left(\frac{k_{5} \cdot k_{2}}{k_{4} \cdot k_{1}}-1\right) C_{14532}-\frac{k_{4} \cdot k_{2}}{k_{2} \cdot k_{3}}\left(\frac{k_{5} \cdot k_{3}}{k_{5} \cdot k_{1}}\right) C_{15432}, \\
& C_{14352}^{\prime}=0, \\
& C_{15342}^{\prime}=0, \\
& C_{14532}^{\prime}=0, \\
& C_{15432}^{\prime}=0 .
\end{aligned}
$$


Since the amplitude is invariant under this color-factor shift, we have

$$
\mathcal{A}_{5,0}=A(13452) C_{13452}^{\prime}+A(13542) C_{13542}^{\prime} .
$$

Substituting eq. (A.7) into eq. (A.8) and equating to eq. (A.1) we obtain

$$
\begin{aligned}
& A(14352)=-\frac{k_{4} \cdot\left(k_{1}+k_{3}\right)}{k_{4} \cdot k_{1}} A(13452)+\frac{k_{4} \cdot k_{2}}{k_{4} \cdot k_{1}} A(13542), \\
& A(15342)=\frac{k_{5} \cdot k_{2}}{k_{5} \cdot k_{1}} A(13452)-\frac{k_{5} \cdot\left(k_{1}+k_{3}\right)}{k_{5} \cdot k_{1}} A(13542), \\
& A(14532)=-\frac{k_{2} \cdot k_{5}}{k_{2} \cdot k_{3}}\left(\frac{k_{4} \cdot k_{3}}{k_{4} \cdot k_{1}}\right) A(13452)+\frac{k_{4} \cdot k_{2}}{k_{2} \cdot k_{3}}\left(\frac{k_{5} \cdot k_{2}}{k_{4} \cdot k_{1}}-1\right) A(13542), \\
& A(15432)=\frac{k_{2} \cdot k_{5}}{k_{2} \cdot k_{3}}\left(\frac{k_{4} \cdot k_{2}}{k_{5} \cdot k_{1}}-1\right) A(13452)-\frac{k_{4} \cdot k_{2}}{k_{2} \cdot k_{3}}\left(\frac{k_{5} \cdot k_{3}}{k_{5} \cdot k_{1}}\right) A(13542)
\end{aligned}
$$

which are precisely the BCJ relations for five-gluon partial amplitudes [10].

An analogous procedure can be used for any $n$-point QCD amplitude to define shifted color factors that vanish except for those belonging to the JO basis.

Open Access. This article is distributed under the terms of the Creative Commons Attribution License (CC-BY 4.0), which permits any use, distribution and reproduction in any medium, provided the original author(s) and source are credited.

\section{References}

[1] H. Kawai, D.C. Lewellen and S.H.H. Tye, A relation between tree amplitudes of closed and open strings, Nucl. Phys. B 269 (1986) 1 [INSPIRE].

[2] F.A. Berends, W.T. Giele and H. Kuijf, On relations between multi-gluon and multigraviton scattering, Phys. Lett. B 211 (1988) 91 [INSPIRE].

[3] Z. Bern, L.J. Dixon, M. Perelstein and J.S. Rozowsky, Multileg one loop gravity amplitudes from gauge theory, Nucl. Phys. B 546 (1999) 423 [hep-th/9811140] [INSPIRE].

[4] N.E.J. Bjerrum-Bohr, P.H. Damgaard, B. Feng and T. Sondergaard, Gravity and Yang-Mills amplitude relations, Phys. Rev. D 82 (2010) 107702 [arXiv: 1005.4367] [INSPIRE].

[5] N.E.J. Bjerrum-Bohr, P.H. Damgaard, B. Feng and T. Sondergaard, New identities among gauge theory amplitudes, Phys. Lett. B 691 (2010) 268 [arXiv:1006.3214] [INSPIRE].

[6] N.E.J. Bjerrum-Bohr, P.H. Damgaard, B. Feng and T. Sondergaard, Proof of gravity and Yang-Mills amplitude relations, JHEP 09 (2010) 067 [arXiv: 1007.3111] [inSPIRE].

[7] N.E.J. Bjerrum-Bohr, P.H. Damgaard, T. Sondergaard and P. Vanhove, The momentum kernel of gauge and gravity theories, JHEP 01 (2011) 001 [arXiv:1010.3933] [INSPIRE].

[8] J. Broedel, O. Schlotterer and S. Stieberger, Polylogarithms, multiple Zeta values and superstring amplitudes, Fortsch. Phys. 61 (2013) 812 [arXiv:1304.7267] [INSPIRE].

[9] F. Cachazo, S. He and E.Y. Yuan, Scattering of massless particles: scalars, gluons and gravitons, JHEP 07 (2014) 033 [arXiv: 1309.0885] [INSPIRE].

[10] Z. Bern, J.J.M. Carrasco and H. Johansson, New relations for gauge-theory amplitudes, Phys. Rev. D 78 (2008) 085011 [arXiv:0805.3993] [INSPIRE]. 
[11] Z. Bern, T. Dennen, Y.-t. Huang and M. Kiermaier, Gravity as the square of gauge theory, Phys. Rev. D 82 (2010) 065003 [arXiv: 1004.0693] [InSPIRE].

[12] R. Monteiro, D. O'Connell and C.D. White, Black holes and the double copy, JHEP 12 (2014) 056 [arXiv: 1410.0239] [INSPIRE].

[13] A. Luna, R. Monteiro, D. O'Connell and C.D. White, The classical double copy for Taub-NUT spacetime, Phys. Lett. B 750 (2015) 272 [arXiv:1507.01869] [INSPIRE].

[14] A. Luna, R. Monteiro, I. Nicholson, D. O'Connell and C.D. White, The double copy: Bremsstrahlung and accelerating black holes, JHEP 06 (2016) 023 [arXiv:1603.05737] [INSPIRE].

[15] A.K. Ridgway and M.B. Wise, Static spherically symmetric Kerr-Schild metrics and implications for the classical double copy, Phys. Rev. D 94 (2016) 044023 [arXiv: 1512.02243] [INSPIRE].

[16] A. Luna et al., Perturbative spacetimes from Yang-Mills theory, JHEP 04 (2017) 069 [arXiv: 1611.07508] [INSPIRE].

[17] Z. Bern, J.J.M. Carrasco and H. Johansson, Perturbative quantum gravity as a double copy of gauge theory, Phys. Rev. Lett. 105 (2010) 061602 [arXiv:1004.0476] [INSPIRE].

[18] J.J. Carrasco and H. Johansson, Five-point amplitudes in $N=4$ super-Yang-Mills theory and $N=8$ supergravity, Phys. Rev. D 85 (2012) 025006 [arXiv:1106.4711] [InSPIRE].

[19] Z. Bern, C. Boucher-Veronneau and H. Johansson, $N>4$ supergravity amplitudes from gauge theory at one loop, Phys. Rev. D 84 (2011) 105035 [arXiv:1107.1935] [INSPIRE].

[20] Z. Bern, J.J.M. Carrasco, L.J. Dixon, H. Johansson and R. Roiban, Simplifying multiloop integrands and ultraviolet divergences of gauge theory and gravity amplitudes, Phys. Rev. D 85 (2012) 105014 [arXiv: 1201.5366] [INSPIRE].

[21] E.Y. Yuan, Virtual color-kinematics duality: 6-pt 1-Loop MHV amplitudes, JHEP 05 (2013) 070 [arXiv:1210.1816] [INSPIRE].

[22] R.H. Boels, B.A. Kniehl, O.V. Tarasov and G. Yang, Color-kinematic duality for form factors, JHEP 02 (2013) 063 [arXiv: 1211.7028] [INSPIRE].

[23] J.J.M. Carrasco, M. Chiodaroli, M. Günaydin and R. Roiban, One-loop four-point amplitudes in pure and matter-coupled $N \leq 4$ supergravity, JHEP 03 (2013) 056 [arXiv: 1212.1146] [INSPIRE].

[24] N.E.J. Bjerrum-Bohr, T. Dennen, R. Monteiro and D. O'Connell, Integrand oxidation and one-loop colour-dual numerators in $N=4$ gauge theory, JHEP 07 (2013) 092 [arXiv: 1303.2913] [INSPIRE].

[25] Z. Bern, S. Davies, T. Dennen, Y.-t. Huang and J. Nohle, Color-kinematics duality for pure Yang-Mills and gravity at one and two loops, Phys. Rev. D 92 (2015) 045041 [arXiv: 1303.6605] [INSPIRE].

[26] Z. Bern, S. Davies, T. Dennen, A.V. Smirnov and V.A. Smirnov, Ultraviolet properties of $N=4$ supergravity at four loops, Phys. Rev. Lett. 111 (2013) 231302 [arXiv:1309.2498] [INSPIRE].

[27] J. Nohle, Color-kinematics duality in one-loop four-gluon amplitudes with matter, Phys. Rev. D 90 (2014) 025020 [arXiv:1309.7416] [INSPIRE]. 
[28] Z. Bern, S. Davies and T. Dennen, Enhanced ultraviolet cancellations in $\mathcal{N}=5$ supergravity at four loops, Phys. Rev. D 90 (2014) 105011 [arXiv: 1409.3089] [InSPIRE].

[29] Z. Bern, J.J. Carrasco, W.-M. Chen, H. Johansson and R. Roiban, Gravity amplitudes as generalized double copies of gauge-theory amplitudes, Phys. Rev. Lett. 118 (2017) 181602 [arXiv: 1701.02519] [INSPIRE].

[30] Z. Bern et al., Five-loop four-point integrand of $N=8$ supergravity as a generalized double copy, Phys. Rev. D 96 (2017) 126012 [arXiv:1708.06807] [inSPIRE].

[31] S. He and O. Schlotterer, New relations for gauge-theory and gravity amplitudes at loop level, Phys. Rev. Lett. 118 (2017) 161601 [arXiv:1612.00417] [INSPIRE].

[32] S. He, O. Schlotterer and Y. Zhang, New BCJ representations for one-loop amplitudes in gauge theories and gravity, arXiv:1706.00640 [INSPIRE].

[33] Z. Bern, A. De Freitas and H.L. Wong, On the coupling of gravitons to matter, Phys. Rev. Lett. 84 (2000) 3531 [hep-th/9912033] [INSPIRE].

[34] Y.-J. Du, B. Feng and C.-H. Fu, BCJ relation of color scalar theory and KLT relation of gauge theory, JHEP 08 (2011) 129 [arXiv:1105.3503] [INSPIRE].

[35] R. Britto, F. Cachazo, B. Feng and E. Witten, Direct proof of tree-level recursion relation in Yang-Mills theory, Phys. Rev. Lett. 94 (2005) 181602 [hep-th/0501052] [INSPIRE].

[36] D.-p. Zhu, Zeros in scattering amplitudes and the structure of nonabelian gauge theories, Phys. Rev. D 22 (1980) 2266 [INSPIRE].

[37] C.J. Goebel, F. Halzen and J.P. Leveille, Angular zeros of Brown, Mikaelian, Sahdev and Samuel and the factorization of tree amplitudes in gauge theories, Phys. Rev. D 23 (1981) 2682 [INSPIRE].

[38] R.W. Brown, K.L. Kowalski and S.J. Brodsky, Classical radiation zeros in gauge theory amplitudes, Phys. Rev. D 28 (1983) 624 [InSPIRE].

[39] R.W. Brown and S.G. Naculich, BCJ relations from a new symmetry of gauge-theory amplitudes, JHEP 10 (2016) 130 [arXiv:1608.04387] [INSPIRE].

[40] R.W. Brown and S.G. Naculich, Color-factor symmetry and BCJ relations for QCD amplitudes, JHEP 11 (2016) 060 [arXiv:1608.05291] [INSPIRE].

[41] R. Kleiss and H. Kuijf, Multi-gluon cross-sections and five jet production at hadron colliders, Nucl. Phys. B 312 (1989) 616 [INSPIRE].

[42] T. Melia, Dyck words and multiquark primitive amplitudes, Phys. Rev. D 88 (2013) 014020 [arXiv: 1304.7809] [INSPIRE].

[43] T. Melia, Getting more flavor out of one-flavor QCD, Phys. Rev. D 89 (2014) 074012 [arXiv: 1312.0599] [INSPIRE].

[44] H. Johansson and A. Ochirov, Color-kinematics duality for QCD amplitudes, JHEP 01 (2016) 170 [arXiv: 1507.00332] [INSPIRE].

[45] B. Feng and S. He, KLT and new relations for $N=8$ SUGRA and $N=4$ SYM, JHEP 09 (2010) 043 [arXiv:1007.0055] [INSPIRE].

[46] P.H. Damgaard, R. Huang, T. Sondergaard and Y. Zhang, The complete KLT-map between gravity and gauge theories, JHEP 08 (2012) 101 [arXiv:1206.1577] [INSPIRE]. 
[47] L. de la Cruz, A. Kniss and S. Weinzierl, Double copies of fermions as matter that interacts only gravitationally, Phys. Rev. Lett. 116 (2016) 201601 [arXiv: 1601.04523] [INSPIRE].

[48] S.G. Naculich, Scattering equations and BCJ relations for gauge and gravitational amplitudes with massive scalar particles, JHEP 09 (2014) 029 [arXiv:1407.7836] [INSPIRE].

[49] A. Anastasiou et al., Twin supergravities from Yang-Mills theory squared, Phys. Rev. D 96 (2017) 026013 [arXiv: 1610.07192] [INSPIRE].

[50] T. Melia, Proof of a new colour decomposition for QCD amplitudes, JHEP 12 (2015) 107 [arXiv: 1509.03297] [INSPIRE].

[51] V. Del Duca, L.J. Dixon and F. Maltoni, New color decompositions for gauge amplitudes at tree and loop level, Nucl. Phys. B 571 (2000) 51 [hep-ph/9910563] [inSPIRE].

[52] D. Kosower, B.-H. Lee and V.P. Nair, Multi gluon scattering: a string based calculation, Phys. Lett. B 201 (1988) 85 [INSPIRE].

[53] M.L. Mangano, The color structure of gluon emission, Nucl. Phys. B 309 (1988) 461 [INSPIRE].

[54] G. Kälin, Cyclic Mario Worlds - Color-decomposition for one-loop QCD, arXiv: 1712.03539 [INSPIRE].

[55] D. Vaman and Y.-P. Yao, Constraints and generalized gauge transformations on tree-level gluon and graviton amplitudes, JHEP 11 (2010) 028 [arXiv: 1007.3475] [INSPIRE].

[56] F. Cachazo, S. He and E.Y. Yuan, Scattering equations and Kawai-Lewellen-Tye orthogonality, Phys. Rev. D 90 (2014) 065001 [arXiv:1306.6575] [INSPIRE].

[57] N.E.J. Bjerrum-Bohr, P.H. Damgaard and P. Vanhove, Minimal basis for gauge theory amplitudes, Phys. Rev. Lett. 103 (2009) 161602 [arXiv:0907.1425] [INSPIRE].

[58] S. Stieberger, Open \& closed vs. pure open string disk amplitudes, arXiv:0907.2211 [INSPIRE].

[59] B. Feng, R. Huang and Y. Jia, Gauge amplitude identities by on-shell recursion relation in S-matrix program, Phys. Lett. B 695 (2011) 350 [arXiv: 1004.3417] [INSPIRE].

[60] Y.-X. Chen, Y.-J. Du and B. Feng, A proof of the explicit minimal-basis expansion of tree amplitudes in gauge field theory, JHEP 02 (2011) 112 [arXiv:1101.0009] [INSPIRE].

[61] L. de la Cruz, A. Kniss and S. Weinzierl, Proof of the fundamental BCJ relations for QCD amplitudes, JHEP 09 (2015) 197 [arXiv: 1508.01432] [INSPIRE].

[62] M. Kiermaier, Gravity as the square of gauge theory, talk given at Amplitudes 2010, May 4-7, Queen Mary University of London, London, U.K. (2010).

[63] F. Cachazo, S. He and E.Y. Yuan, Scattering equations and matrices: from Einstein to Yang-Mills, DBI and NLSM, JHEP 07 (2015) 149 [arXiv:1412.3479] [INSPIRE].

[64] H. Johansson and A. Ochirov, Pure gravities via color-kinematics duality for fundamental matter, JHEP 11 (2015) 046 [arXiv:1407.4772] [INSPIRE].

[65] M. Chiodaroli, M. Günaydin, H. Johansson and R. Roiban, Scattering amplitudes in $\mathcal{N}=2$ Maxwell-Einstein and Yang-Mills/Einstein supergravity, JHEP 01 (2015) 081 [arXiv: 1408.0764] [INSPIRE]. 